\title{
A SÍNTESE DA TEORIA GERAL \\ DO EMPREGO, DOS JUROS E DA MOEDA \\ SEGUNDO ROY HARROD EM \\ "Mr. KEYNES AND TRADITIONAL THEORY"*
}

Claudia Heller**

\section{PreÂMbULO}

Entre as primeiras interpretações formalizadas da Teoria Geral do Emprego, dos Juros e da Moeda, de John Maynard Keynes, estão os textos apresentados por Roy HARROD, John HICKS e James MEADE no Simpósio "Mr. Keynes System", durante a Sexta Conferência Européia da Sociedade de Econometria, realizado em Oxford, de 25 a 29 de setembro de 1936. Os trabalhos de HARROD ("Mr. Keynes and Traditional Theory") e de HICKS ("Mr. Keynes and the 'Classics': a suggested interpretation") foram posteriormente publicados na revista Econometrica em janeiro e em abril de 1937, respectivamente. O de MEADE (“A simplified model of Mr. Keynes' system”) foi publicado pela Review of Economic Studies, em fevereiro de 1937. O relatório do encontro, por sua vez foi escrito por PHELPS-BROWN e publicado em Econometrica, em outubro de $1937 .{ }^{1}$

*Preparado para o IV Encontro Nacional de Economia Política, 1 a 4 de junho de 1999, Porto Alegre. Devo agradecimentos a Estela Alvarez Ribeiro, que, durante a elaboração de sua monografia de conclusão do Curso de Ciências Econômicas na FCLAr/UNESP, não apenas foi de grande auxílio na pesquisa bibliográfica, mas também um estímulo para desenvolver e aprofundar esta pesquisa.

***Professora do Departamento de Economia, Faculdade de Ciências e Letras da Universidade Estadual Paulista (FCLAr/UNESP), Araraquara. hellerc@ fclar.unesp.br

1 Ver PHELPS-BROWN, E. H. Report of the Oxford Meeting, September 25-29 1936. Econometrica, v. 5, p. 361-383, Oct. 1990. 
O único trabalho que, com certeza, não circulou antes do evento foi o de HICKS. Entretanto, HICKS teve a oportunidade de ler o de HARROD e o de MEADE antes de terminar o seu, e este é um dos motivos - mas não o único - pelos quais YOUNG (1987) sustenta que foram os sistemas de equações de HARROD e de MEADE que inspiraram o modelo matemático (e gráfico) elaborado por HICKS - o qual veio a ser conhecido como o modelo IS-LM. ${ }^{2}$

Aparentemente, os textos publicados como textos apresentados no encontro não correspondem exatamente aos que foram divulgados no próprio encontro nem aos que circularam antes do evento - ou seja, são versões revistas. YOUNG (1987) registra que entre a primeira resenha da Teoria Geral feita por HICKS, em 1936, e o texto publicado como o apresentado no encontro, em 1937, houve uma versão intermediária, que foi enviada por HICKS a Keynes em 16 de outubro de $1936 .{ }^{3}$ Esta versão, segundo MOGGRIDGE (1973/1987, p. 77, n. 1), não sobreviveu e YOUNG (1987, p. 31-32) insinua que a famosa (e controvertida) concordância de Keynes com relação à interpretação da Teoria Geral de HICKS pode referir-se à versão desaparecida do trabalho de HICKS, e não ao artigo efetivamente publicado em 1937. A existência de uma versão anterior é confirmada por HICKS no próprio artigo de 1937, no qual informa que o texto apresentado na conferência gerou um debate interessante, tendo sido subseqüentemente modificado "em parte à luz deste debate, e em parte como resultado de novas discussões em Cambridge.” (HICKS, 1937, p. 147, n. 1)

Quanto a HARROD, também há indicações de que seu artigo de 1937 foi precedido de um outro, que, como é afirmado na literatura, circulou em Cambridge, em 1936, na forma mimeografada (e que não é sua resenha da Teoria Geral, publicada em Political Quarterly em abril de 1936). HARROD enviou a Keynes uma versão do trabalho que seria apresentado na Conferência de Oxford e Keynes convidou-o a publicá-lo no Economic Journal. HARROD comprometeu-se a rever o trabalho, mas este acabou sendo publicado em Econometrica, em janeiro de 1937. PATINKIN (1990, p. 212) sugere que o convite de Keynes se referia ao rascunho de HARROD, preparatório para o Encontro de Oxford, não necessariamente idêntico ao que foi publicado em 1937.4

$2 \mathrm{O}$ termo utilizado originalmente por HICKS foi IS-LL (ou SILL). O nome IS-LM foi cunhado posteriormente por Hansen.

3 A resenha intitulava-se originalmente "Mr. Keynes's theory of employment" e foi publicada em 1936 no The Economic Journal. Quando reproduzida numa coletânea de trabalhos de HICKS, publicada em 1982, recebeu o título de "The General Theory: a first impression".

4 Veja também MOGGRIDGE, D. John Maynard Keynes: the General Theory and after - Parte II: defence and development. London: Macmillan, 1973/1987. (The collected writings of John Maynard Keynes - CWJMR, 14). p. 83, n. 1. 
No que se refere a MEADE, YOUNG (1987, p. 33-37) encontra indícios de que tampouco o texto publicado na Review of Economic Studies é o mesmo que foi apresentado na conferência. Não se sabe ao certo se MEADE teve ou não acesso ao texto de HARROD, nem se o texto de MEADE circulou antes do encontro de Oxford, mas existe registro de que Keynes o havia lido previamente. YOUNG (1987, p. 37-38) chama a atenção para o fato de que só o de MEADE não foi publicado em Econometrica e considera particularmente estranha a rejeição desse artigo pelo Economic Journal. ${ }^{5}$

O que parece ser fato é que Keynes e HICKS tiveram a oportunidade de ler os trabalhos de HARROD e de MEADE antes que eles fossem apresentados na conferência e que Keynes recebeu uma cópia do artigo de HICKS em outubro de 1936, logo após o evento.

Outros trabalhos que representam as primeiras formalizações da Teoria Geral são os de Brian REDDAWAY (The General Theory of Employment, Interest and Money) e de David CHAMPERNOWNE (Unemployment, Basic and Monetary: the Classical Analysis and the Keynesian), publicados antes da Conferência de Oxford, da qual não participaram. A resenha de REDDAWAY foi publicada num periódico australiano - o Economic Record - e o artigo de CHAMPERNOWNE foi publicado na Review of Economic Studies, ambos em junho de 1936. Chama a atenção, entretanto, o fato de que, em alguns aspectos importantes, seus artigos são bastante semelhantes aos de HARROD, HICKS e de MEADE: ambos usaram sistemas de equações simultâneas (e no caso de CHAMPERNOWNE, também diagramas) para descrever o que consideravam ser os princípios centrais da teoria "clássica" e da Teoria Geral. ${ }^{6}$

$5 \mathrm{O}$ registro de que Keynes havia lido o artigo de MEADE antes da conferência encontra-se num cartão datado de 14 de setembro de 1936, enviado por Keynes a MEADE. A esse respeito, ver YOUNG, W. Interpreting Keynes: The IS/LM Enigma. Boulder: Westview Press; Oxford: Basil Blackwell, 1987. p. 34; PATINKIN, D. Or different interpretations of "The General Theory”. Journal of Monetary Economics, v. 26, p. 205-243, Oct. 1990; SKIDELSKY, R. John Maynard Keynes - The economist as savior 1920-1937. London: Macmillan, 1992. p. 614; e BARENS, I. From Keynes to Hicks - an aberration? IS-LM and the analytical nucleus of the general theory. Paper present at the Second Annual Conference of the European Society for the History of Economic Thought, Bologna, 27/02 - 1/03/1998. Mineog. To be published in ANTONI, E. di; HOWITT, P.; LEIJONHUFVUD, A. (Ed.). Money, marrets and method: essays in honor of Robert Clower. Chletenhna: Edward Elgar, 1998. p. 38, n. 72. Econometrica publicou apenas um resumo do trabalho de MEADE, incorporado ao relatório da conferência, escrito por PHELPS-BROWN. Ver PHELPS-BROWN, op. cit., p. 363.

6 Segundo YOUNG, op. cit., p. 199-200, n. 11, eles assim o fizeram inspirados nas aulas de Keynes (de quem foram alunos), dadas no período 1932-34. Veja também PATINKIN, op. cit., p. 234, n. 29. 
Ainda segundo YOUNG (1987, p. 82-83), o fato de que HICKS fazia parte da comissão editorial de Review of Economic Studies na época em que CHAMPERNOWNE submeteu seu trabalho para publicação indica a possibilidade de que HICKS também tenha se utilizado do trabalho de CHAMPERNOWNE para elaborar o seu.

Em síntese: antes de escrever seu artigo, HICKS leu os trabalhos de MEADE e de HARROD, e possivelmente também o de CHAMPERNOWNE. Não há registro de que o de REDDAWAY fosse conhecido por HICKS ou pelos demais participantes da conferência, mas, como foi publicado em junho de 1936, esta hipótese não pode ser descartada. MEADE, aparentemente, escreveu o seu sem ter lido o de HARROD e certamente sem ter lido o de HICKS, e HARROD não leu o de HICKS, mas pode ter lido o de MEADE. Estes elementos históricos e a análise comparativa do conteúdo de cada um levaram YOUNG (1987, p. 169) a concluir que

\begin{abstract}
a abordagem da IS-LM é primeiramente um produto da descoberta de um sistema de equações por Harrod e Meade [...] Depois, baseado no sistema de equações Harrod-Meade, veio a descoberta crucial de Hicks, de uma maneira de representar este sistema de equações por meio de diagramas [...] na sua agora famosa 'sugestão de interpretação’ da Teoria Geral de Keynes.
\end{abstract}

Por este motivo, YOUNG (1987, p. 173) sugere que esta interpretação deve ser denominada como "IS-LM de HARROD-HICKS-MEADE".

Outros autores, como BARENS (1998), endossam a conclusão de YOUNG, mas o fazem apenas parcialmente. Na sua opinião, "pode-se dizer que foi Meade quem inventou o modelo IS-LM da Teoria Geral, e que Hicks adicionou o diagrama para representar a solução pela geometria" (BARENS, 1998, p. 24-25). Em seu endosso, BARENS não faz qualquer referência a HARROD, REDDAWAY ou CHAMPERNOWNE. Os trabalhos de HARROD e REDDAWAY só são mencionados para efeito de comparação entre as reações de Keynes a cada um desses trabalhos, e CHAMPERNOWNE não consta nem mesmo da sua bibliografia.

7 Vale ressaltar que YOUNG não atribui o modelo às equações simultâneas de CHAMPERNOWNE e de REDDAWAY (nem aos diagramas de CHAMPERNOWNE), embora considere que eles também fornecem as bases para um modelo IS-LM. A este respeito vejase também DARITY JR., W.; YOUNG, W. IS-LM: an inquest. History of Political Economy, v. 27, n. 1, p. 1-41, 1995. 


\section{INTRODUÇÃO}

O breve relato feito no Preâmbulo constitui a base sobre a qual tem crescido o número de autores que atribuem a primeira formulação matematizada e diagramática da Teoria Geral a um processo de "fertilização cruzada" entre HARROD, HICKS e MEADE - incluindo, eventualmente, CHAMPERNOWNE e REDDAWAY. Essas interpretações sobre a origem da IS-LM têm procurado sustentação não apenas na história documental do período imediato posterior à publicação da Teoria Geral, mas também na comparação analítica das contribuições desses autores. Essas interpretações, entretanto, têm enfatizado demasiadamente a semelhança formal entre os sistemas de equações simultâneas propostos, ao mesmo tempo em que negligenciam os variados argumentos sobre os quais os modelos formais foram construídos. Ademais, de um modo geral, concluem que o sucesso da formalização da Teoria Geral se deve à elegância matemática e ao caráter preciso dos modelos baseados em equações simultâneas, que consideram semelhantes uns aos outros e, em particular, atribuem o sucesso de aceitação da versão de HICKS ao fato de ele ter sido o único a representar a teoria por meio de diagramas. ${ }^{8}$

Este texto tem por objetivo explorar a hipótese de que, embora se possa aceitar que o sucesso dos modelos formais da Teoria Geral decorra da preferência dos economistas por modelos caracterizados por serem "determinados, simétricos e sem incerteza" (YOUNG, 1987, p. 82), deve-se levar em conta também, e talvez principalmente, o fato de que os argumentos que os sustentam permitem a convivência simultânea de variadas relações de causalidade. A despeito das semelhanças, no que se refere à forma final das equações representativas de cada "teoria" (clássica ou de Keynes) descritas pelos autores mencionados no Preâmbulo, as relações de causalidade e os argumentos utilizados para "transformar", "inverter", "complementar" e/ou "generalizar" estas equações foram, muitas vezes, bastante diferentes. ${ }^{9}$

8 Ver, por exemplo, BEAUD, M.; DOSTALER, G. Economic thought since Keynes - a history and dictionary of major economists. London: Routledge, 1995. p. 81.

9 Neste trabalho, o uso da designação "clássicos" - ou teoria "tradicional", "vigente" ou ainda "ortodoxa" - é propositadamente genérico. Assume-se como "clássico" ou qualquer outra destas variantes, os sistemas que os próprios autores tratados consideram como tal, sem fazer distinção entre os termos. Do mesmo modo, as expressões "matematização", "formalização", "uso de equações" e/ou de "funções matemáticas" designam simplesmente o uso de representação simbólica típica da matemática. Também a expressão "diagramas" designa 
Por razões de tempo e de espaço, este texto cinge-se apenas à análise da formalização elaborada por HARROD. A razão "tempo" deriva do fato de este texto constituir-se no resultado parcial de uma pesquisa mais ampla (e ainda inconclusa), que visa dar conta de uma parte do debate ocorrido nos primeiros anos que se seguiram à publicação da Teoria Geral, caracterizado pela ênfase na comparação entre a teoria de Keynes e a teoria vigente (também chamada de "ortodoxa", "clássica" ou "tradicional") e a tentativa de compatibilizá-las, às vezes através de alguma sistematização matemática e/ou diagramática. A razão "espaço" decorre do fato de que, dos textos mencionados no Preâmbulo, o mais lido, debatido e analisado é, sem dúvida alguma, o de HICKS, enquanto que os de MEADE, CHAMPERNOWNE e REDDAWAY, embora menos conhecidos, são relativamente simples e não muito controversos. O texto de HARROD, entretanto, caracteriza-se por ser complexo e relativamente pouco conhecido. Consequentemente sua análise não pode ser feita de modo apressado, especialmente quando se pretende destacar os argumentos e os raciocínios utilizados pelo autor para construir seu sistema de equações representativas da Teoria Geral.

É importante observar que este trabalho não se propõe a discutir a(s) (in)correção(ções) da descrição feita por HARROD, seja da teoria de Keynes, seja da visão teórica que Keynes combatia. O trabalho visa tão-somente considerar os argumentos de caráter teórico que sustentam o resultado formal encontrado por HARROD, de modo a subsidiar a discussão mais geral - a ser concluída posteriormente - dos motivos pelos quais a sistematização da Teoria Geral, por meio da matemática, foi tão bem aceita. Neste sentido, é importante esclarecer que este trabalho, embora tratando apenas da versão da Teoria Geral elaborada por HARROD, é uma primeira aproximação - necessária, porém insuficiente - para a questão mais ampla, que trata dos motivos do sucesso da "síntese neoclássica". ${ }^{10}$

a utilização de gráficos independentemente da precisão geométrica ou trigonométrica. Neste aspecto, seguimos e ampliamos a sugestão de O'DONNEL (1997, p. 132), que, ao explicitar o seu objeto de estudo - a opinião de Keynes sobre o uso do formalismo em economia, explica: "por formalismo entendo a representação simbólica, a matemática, a inferência estatística ou a econometria”. Cf. O'DONNEL, R. Keynes and formalism. In: HARCOURT, G. C.; RIACH, R. A. A "second edition" of the General Theory. London: Routledge, 1997. v. 2, p. 131-165.

10 A hipótese discutida na pesquisa ainda inconclusa é a de que as formalizações matemáticas elaboradas por HARROD, HICKS e MEADE, além das de CHAMPERNOWNE e de REDDAWAY, ainda que semelhantes na forma final, foram alcançadas mediante diferentes raciocínios, justificativas e argumentos teóricos, e que a aceitação e o sucesso da versão matematizada se deu pelo fato de ela permitir a incorporação - de modo implícito - dessas variadas relações de causalidade. 


\section{A SÍNTESE DE HARROD ${ }^{11}$}

Para HARROD, a teoria econômica divide-se em uma parte que é
geral e outra que é composta de "ramos especializados". A parte geral

[...]compõe-se basicamente de um número de equações funcionais que expressam esquemas de preferências individuais, de um número de identidades [...] e elucida questões tais como se o número de equações é igual ao número de incógnitas, ou se as soluções são únicas ou múltiplas. A resposta a estas perguntas deve esclarecer se o equilíbrio do sistema como um todo é estável, instável ou indeterminado, se há posições alternativas de equilíbrio etc. Deve haver indícios sobre as formas genéricas de algumas das equações funcionais [...] (HARROD, 1937, p. 74)

A parte dos "ramos especializados", por sua vez, lida com problemas específicos, e seu método consiste em "assumir que certos termos, que aparecem como variáveis no sistema geral de equações, podem ser tratados como constantes para o propósito especial em questão" (HARROD, 1937, p. 75). A estes métodos inerentes aos "ramos especializados" HARROD denomina "atalhos".

O mais importante dos "ramos especializados" tratados por Keynes na concepção de HARROD - refere-se ao problema dos juros. Na teoria tradicional, esse "ramo especializado" é tratado por meio de duas equações e duas incógnitas. As equações são as de "demanda e oferta relativas a um bem em particular" (HARROD, 1937, p. 75) - respectivamente: $y=f(x)$ e $x=f(y)$. A equação de demanda diz que " $\mathrm{y}$, a produtividade marginal do capital, depende de $\mathrm{x}$, o montante de capital investido por unidade de tempo" (HARROD, 1937, p. 76) e a de oferta

11 Conforme mencionado anteriormente, não se pretende avaliar o "grau de fidelidade" com que HARROD interpretou a teoria "clássica" e/ou a "teoria de Keynes". O que se objetiva é identificar os argumentos teóricos e econômicos subjacentes à sua formulação matemática, destacando o raciocínio que subsidia esta formulação. A exposição do artigo de HARROD explicitará os símbolos originais utilizados e será acompanhada de uma "notação alternativa" que permita uma posterior comparação final entre sua contribuição e a dos demais autores mencionados no Preâmbulo. Serão utilizados, na "notação alternativa", os seguintes símbolos: S para poupança, I para investimento; $r$ para taxa de juros; emc para eficiência marginal do capital, M para moeda; Y para renda, L para preferência pela liquidez, p para preços, w para taxa nominal de salário. O sobrescrito " $\mathrm{S}$ " indica oferta e o sobrescrito " $\mathrm{D}$ " indica demanda. Além disso, serão utilizados os símbolos MDT, MDP e MDE para descrever, respectivamente, a demanda de moeda para transação, por precaução e para especulação. 
HELLER, C. A síntese da teoria geral do emprego,...

diz que " $x$, o montante que os indivíduos escolhem poupar, que é igual ao investimento, depende da taxa de juros" (HARROD, 1937, p. 76). Estas duas equações são suficientes, no escopo da "teoria tradicional", para determinar o valor das duas incógnitas: a taxa de juros e o volume de poupança e, segundo o autor, "não é necessário [...] considerar as controvérsias em torno das formas destas equações" (HARROD, 1937, p. 76), particularmente a que se relaciona aos efeitos de uma elevação da taxa de juros sobre a poupança. ${ }^{12}$

O quadro a seguir descreve a "teoria tradicional" segundo HARROD. Para facilitar a comparação posterior com os demais autores e também porque HARROD usou uma notação muito diferente de todos os demais, é proposta, na penúltima coluna, uma notação alternativa; a última coluna serve apenas para numerar e identificar as equações de HARROD.

Quadro 1 - TEORIA TRADICIONAL, NOTAÇÃO ORIGINAL

\begin{tabular}{|c|c|c|}
\hline & notação alternativa & $\begin{array}{c}\text { Ha: HARROD } \\
\text { C: Clássicos }\end{array}$ \\
\hline $\mathrm{y}=\mathrm{f}(\mathrm{x})^{13}$ & $\mathrm{emc}=\mathrm{f}_{1}(\mathrm{I})$ & $\mathrm{HaC} 1$ \\
\hline $\mathrm{x}=\phi(\mathrm{y})^{14}$ & $\mathrm{~S}=\mathrm{f}_{2}(\mathrm{r})$ ou I $=\mathrm{f}_{2}(\mathrm{r})^{15}$ & $\mathrm{HaC} 2$ \\
\hline $\mathrm{y}=\mathrm{y}^{\prime 16}$ & $\mathrm{emc}=\mathrm{r}$ & $\mathrm{HaC} 3$ \\
\hline
\end{tabular}

Fonte: HARROD, 1937.

A "teoria tradicional", segundo HARROD, caracteriza-se pelo fato de que as incógnitas taxa de juros e poupança são determinadas considerando-se que

12 As definições dadas por HARROD são aparentemente confusas: na equação de demanda, o símbolo [y] representa a produtividade marginal do capital, mas na equação de oferta o mesmo símbolo representa a taxa de juros. Além disso, na equação de demanda, o símbolo [x] representa o montante de capital investido por unidade de tempo e, na equação de oferta, ele representa o montante que os indivíduos escolhem poupar (que é igual ao investimento). Entretanto, a confusão desaparece se se levar em conta que HARROD explicita claramente que "investir-se-á tanto capital de modo que sua produtividade marginal seja igual à taxa de juros, isto é, $y=y^{\prime}$, onde y' é a taxa de juros" (HARROD, 1937, p. 76) o que, segundo o autor, corresponde tanto à teoria tradicional quanto à de Keynes. Além disso, na sua própria definição da equação de oferta, afirma que o montante que os indivíduos escolhem poupar é igual ao investimento.

13 "a produtividade marginal do capital depende do montante de capital investido por unidade de tempo.” (HARROD, 1937, p. 76)

14 "O montante que as pessoas escolhem poupar, que é igual ao montante de investimento, depende da taxa de juros." (HARROD, 1937, p. 76)

$15 \mathrm{I}=\mathrm{S}$ por definição.

16 "investe-se capital até que sua produtividade marginal iguale a taxa de juros."

(HARROD, 1937, p. 76) 
o nível de renda é dado e invariável. Trata-se, portanto, de um "atalho". ${ }^{17} \mathrm{Na}$ teoria de Keynes, entretanto, este "atalho" não pode ser utilizado, pois nela "o ponto isolado mais importante é a visão de que é ilegítimo considerar-se que o nível de renda de uma comunidade é independente do investimento" (HARROD, 1937, p. 76). Portanto, para descrever a Teoria Geral, é preciso incluir, no sistema de equações, uma equação adicional que permita determinar a renda em função do investimento.

Segundo HARROD, a primeira equação da teoria tradicional [HaC1] também é válida para Keynes, pois embora ela afirme que "a produtividade marginal do capital depende do montante de capital investido por unidade de tempo" (ou mais resumidamente: "a produtividade marginal do capital é uma função do investimento"), também pode ser lida como "a eficiência marginal do capital é uma função do investimento" (HARROD, 1937, p. 76), já que "o termo produtividade marginal do capital aparece no livro de Keynes sob o título de eficiência marginal [e] não parece haver uma diferença de princípio [entre os dois termos]" (HARROD, 1937, p. 76-77). HARROD reconhece que Keynes analisa a eficiência marginal de modo "exaustivo e interessante e demonstra que seu valor depende das expectativas empresariais" (HARROD, 1937, p. 77). Considera também que a ênfase dada por Keynes à questão das expectativas não é apenas "sólida, mas constitui um grande aperfeiçoamento da definição de produtividade marginal" e, embora afirme que "este aprimoramento pode ser incorporado à teoria tradicional sem provocar modificações importantes nas outras partes" (HARROD, 1937, p. 77), não o faz.

A segunda equação [HaC2], que na teoria tradicional representa que "o montante que as pessoas escolhem poupar, que é igual ao montante de investimento, depende da taxa de juros" (HARROD, 1937), é modificada para incorporar a renda. Ou seja, a segunda equação, que tinha a forma $\mathrm{x}=\phi(\mathrm{y})$ passa a ter a forma $\mathrm{x}=\phi(\mathrm{y}, \mathrm{i})$ - na notação alternativa, a forma $\mathrm{S}=\mathrm{f}_{2}(\mathrm{r})$ assume a forma $\mathrm{S}=\mathrm{f}_{3}(\mathrm{r}, \mathrm{Y})$ - e indica que "o montante de poupança depende não apenas da taxa de juros, mas também do nível de renda da comunidade" (HARROD, 1937, p. 77). O argumento é o de que "as pessoas poupam uma parcela absoluta maior de uma renda maior [embora] a relação entre o montante que as pessoas escolhem poupar e a taxa de juros seja assunto controverso.” (HARROD, 1937, p. 78)

17 Não é exagerado lembrar que o "atalho" é, segundo o autor, o método inerente à parte dos "ramos especializados" da teoria econômica, que lidam com problemas específicos. O "método" do atalho consiste em supor que alguns dos termos que são variáveis no sistema geral de equações podem ser considerados e tratados como constantes nos ramos especializados. 
Mas, para levar em conta "o ponto isolado mais importante da teoria de Keynes" (HARROD, 1937, p. 76) de que o nível de renda de uma comunidade depende do investimento, HARROD propõe que se utilize esta segunda equação na sua "forma transposta", isto é, $\mathrm{i}=\Psi(\mathrm{x}, \mathrm{y})$, que significa que "o nível de renda depende do montante de investimento (= poupança) e da taxa de juros" (HARROD, 1937, p. 77). Na notação alternativa, a equação original $S=f_{3}(r, Y)$ se transpõe na equação $\mathrm{Y}=\mathrm{f}_{4}(\mathrm{I}, \mathrm{r})$.

Para HARROD, a vantagem desta forma "transposta" da segunda equação é que ela representa a "doutrina do multiplicador", isto é, "a recíproca da fração que expressa a proporção de qualquer renda dada que, a uma dada taxa de juros, as pessoas consumirão" (HARROD, 1937, p. 77). Assim, dado o valor do multiplicador para cada taxa de juros e nível de renda, pode-se, segundo HARROD, deduzir o nível da renda diretamente do volume de investimento. HARROD reconhece que esta sua definição do multiplicador é diferente da de Keynes, que é mais simples e é descrita por $\mathrm{i}=\Psi(\mathrm{x})$, isto é, sem incluir a taxa de juros. Segundo HARROD, Keynes "caiu na tentação de perder a taxa de juros de vista para o bem de um atalho ainda mais curto" (HARROD, 1937, p. 78), mas justifica esta redução do "atalho": em primeiro lugar, porque embora a relação entre o nível de renda e o montante de investimento seja clara, a relação entre a taxa de juros e a poupança é controversa. ${ }^{18}$ Em segundo lugar, a não inclusão da taxa de juros na "doutrina do multiplicador" se explica pelo fato de que, na teoria de Keynes, o montante que as pessoas escolhem poupar é muito mais afetado pelo nível de renda do que pela taxa de juros. Mas considera que "não é preciso brigar por isso [pois] a taxa de juros pode ser recolocada nesta parte do quadro sem afetar o argumento principal", ou seja, que "a propensão a consumir pode ser vista como dependente da taxa de juros, embora, para o bem da simplificação e clareza não se precise insistir neste ponto a cada exposição da doutrina do multiplicador" (HARROD, 1937, p. 78).

Afirmou-se acima que, para HARROD, a teoria econômica divide-se em uma parte que é geral e outra que é composta de "ramos especializados". Segundo o autor, quando se considera que o nível de renda é uma variável a ser determinada (e não mais um "dado"), é preciso incluir uma equação adicional. Esta, na teoria de Keynes (segundo HARROD), é uma equação que se refere ao mercado monetário, descrita por $\mathrm{y}=\chi(\mathrm{m})$. Esta equação representa a preferência pela liquidez e nela "m é a quantidade de moeda, um termo conhecido, dependente da política bancária" (HARROD, 1937, p. 78). HARROD sugere que a renda também seja considerada nesta equação, "uma vez que o montante

18 Note-se que HARROD já havia feito esta observação, ao afirmar que não era preciso discutir a forma das equações de demanda e oferta da "teoria tradicional". 
de moeda requerido pelos consumidores e comerciantes para a circulação ativa depende do nível de renda" (HARROD, 1937, p. 78). Assim, a terceira equação passa a ser $\mathrm{y}=\chi(\mathrm{m}, \mathrm{i}){ }^{19}$

As três equações que acabamos de discutir descrevem o sistema da teoria de Keynes segundo HARROD e estão organizadas no quadro II a seguir.

Quadro 2 - TEORIA DE KEYNES, NOTAÇÃO ORIGINAL

\begin{tabular}{|c|c|c|}
\hline & notação alternativa & $\begin{array}{c}\text { Ha: HARROD } \\
\text { K: Keynes }\end{array}$ \\
\hline$y=f(x)^{20}$ & emc $=f_{1}(I)$ & $\mathrm{HaK} 1$ \\
\hline $\begin{array}{c}\mathrm{x}=\phi(\mathrm{y}, \mathrm{i})^{21} \\
\text { transposta para } \\
\mathrm{i}=\Psi(\mathrm{x}, \mathrm{y})^{22} \\
\text { simplificada para } \\
\mathrm{i}=\Psi(\mathrm{x})^{23}\end{array}$ & $\begin{array}{c}\mathrm{S}=\mathrm{f}_{3}(\mathrm{r}, \mathrm{Y}) \\
\text { transposta para } \\
\mathrm{Y}=\mathrm{f}_{4}(\mathrm{I}, \mathrm{r}) \text { ou } \mathrm{Y}=\mathrm{f}_{4}(\mathrm{~S}, \mathrm{r}) \\
\text { simplificada para } \\
\mathrm{Y}=\mathrm{f}_{5}(\mathrm{I}) \text { ou } \mathrm{Y}=\mathrm{f}_{5}(\mathrm{~S})\end{array}$ & $\begin{array}{l}\mathrm{HaK} 2 \mathrm{a} \\
\mathrm{HaK} 2 \mathrm{~b} \\
\mathrm{HaK} 2 \mathrm{c}\end{array}$ \\
\hline $\begin{array}{c}\mathrm{y}=\chi(\mathrm{m})^{24} \\
\text { ampliada para } \\
\mathrm{y}=\chi(\mathrm{m}, \mathrm{i})^{25}\end{array}$ & $\begin{array}{c}\mathrm{r}=\mathrm{f}_{6}\left(\mathrm{M}^{\mathrm{S}}\right) \\
\text { ampliada para } \\
\mathrm{r}=\mathrm{f}_{7}\left(\mathrm{M}^{\mathrm{S}}, \mathrm{Y}\right)\end{array}$ & $\begin{array}{l}\text { HaK3a } \\
\text { HaK3b }\end{array}$ \\
\hline$y=y^{\prime 26}$ & $\mathrm{emc}=\mathrm{r}$ & $\mathrm{HaK} 4$ \\
\hline
\end{tabular}

Fonte: HARROD, 1937.

19 É interessante notar que, nesta terceira equação, o símbolo [y] que representava a produtividade marginal do capital (ou a eficiência marginal do capital) passa a representar a taxa de juros. Portanto, HARROD mantém a condição de equilíbrio segundo a qual a eficiência marginal do capital iguala a taxa de juros.

20 "a eficiência marginal do capital é uma função do investimento" (HARROD, 1937, p. 76), sem considerar, na formulação, que "o valor [da eficiência marginal] depende das expectativas empresariais” (HARROD, 1937, p. 77). Portanto, a equação HaC1 é igual à equação HaK1.

21 "o montante de poupança depende não apenas da taxa de juros, mas também do nível de renda da comunidade.” (HARROD, 1937, p. 77)

22 "o nível de renda depende do montante de investimento (= poupança) e da taxa de juros.” (HARROD, 1937, p. 77)

23 "[Keynes] caiu na tentação de perder a taxa de juros de vista [na doutrina do multiplicador] para o bem de um atalho ainda mais curto." (HARROD, 1937, p. 78)

24 "este é o esquema da preferência pela liquidez [...] em que m, a quantidade de moeda, é um termo conhecido e depende da política monetária.” (HARROD, 1937, p. 78)

25 "o nível de renda deve ser incluído na equação, uma vez que o montante de moeda requerido pelos consumidores e comerciantes para a circulação ativa depende do nível de renda." (HARROD, 1937, p. 78)

26 "investe-se capital até que sua produtividade marginal iguale a taxa de juros." (HARROD, 1937, p. 76) 
É preciso chamar a atenção para o fato de que, embora HARROD tenha realmente escrito a função $\mathrm{y}=\chi(\mathrm{m})$ e defendido a inclusão da renda nesta função (veja equações HaK3a e HaK3b), seu argumento literal é o de que "o montante de moeda requerido pelos consumidores e comerciantes para a circulação ativa depende do nível de renda" (HARROD, 1937, p. 78). Entende-se aqui que a maneira correta de expressar matematicamente este seu argumento para a inclusão da renda nesta equação seria algo como $\mathrm{M}_{\mathrm{T}}^{\mathrm{D}}=\mathrm{f}_{8}(\mathrm{Y})$ (na notação alternativa esta será a equação HaK3c). Será observado, mais à frente, que na verdade HARROD incluiu em seu sistema uma equação deste tipo. ${ }^{27}$

Embora não haja espaço para desenvolver o argumento, é preciso observar que intérpretes que desconsideram os raciocínios presentes nos textos originais - como o que se acabou de destacar - e enfatizam a formulação matemática (por vezes incompleta ou até incorreta, como já foi visto), encontram mais semelhanças do que as que de fato existem entre HARROD, HICKS e MEADE.

$\mathrm{Na}$ "teoria de Keynes", segundo HARROD, as incógnitas são a taxa de juros [r], a poupança [S] e o nível de renda [Y] - que não é mais considerado dado. Entretanto, ao expor o raciocínio pelo qual se determinam os valores destas incógnitas, HARROD procede a novas modificações dessas relações, compondo um sistema diferente e mais ampliado. $\mathrm{O}$ autor afirma textualmente que os resultados anteriores podem ser resumidos como segue:

[...] temos agora três equações para determinar o valor de três incógnitas - o nível de renda, o volume de poupança (= investimento) e a taxa de juros (= eficiência marginal do capital) [...] O montante de investimento (= montante de poupança) depende da produtividade marginal do capital e da taxa de juros. O nível de renda está relacionado ao montante de investimento através do multiplicador, isto é, pela propensão a consumir; e a taxa de juros depende do desejo por reservas líquidas e do montante de moeda disponível para satisfazer este desejo. Este montante de moeda disponível depende da política dos bancos ao determinarem o valor de suas dívidas a receber e do nível de renda (quanto maior o nível de renda, mais moeda será necessária para a circulação ativa)." (HARROD, 1937, p. 79)

Uma possível descrição formal destas relações é a que se propõe no quadro a seguir. ${ }^{28}$

27 Trata-se da equação HaG5 no quadro 4, proposto na conclusão.

28 A formalização matemática sugerida não consta do original e por este motivo a notação utilizada corresponde apenas a uma sugestão que dá sequiência à "notação alternativa" dos quadros anteriores. 


\begin{tabular}{|l|c|c|}
\hline & notação sugerida & $\begin{array}{c}\text { Ha: HARROD } \\
\text { R: Keynes Resumido }\end{array}$ \\
\hline $\begin{array}{l}\text { "O montante de investimento }(= \\
\text { montante de poupança) depende da } \\
\text { produtividade marginal do capital e da } \\
\text { taxa de juros" }\end{array}$ & ${\mathrm{I}=\mathrm{f}_{9}(\mathrm{emc}, \mathrm{r})}^{\text {HaR1 }}$ \\
\hline $\begin{array}{l}\text { "O nível de renda está relacionado ao } \\
\text { montante de investimento através do } \\
\text { multiplicador, isto é, pela propensão a } \\
\text { consumir" }\end{array}$ & $\begin{array}{c}\mathrm{Y}=\mathrm{f}_{4}(\mathrm{I}, \mathrm{r}) \text { ou } \mathrm{Y}=\mathrm{f}_{5}(\mathrm{I}) \\
\mathrm{f}_{5} \text { representa a propensão } \\
\text { marginal a consumir }\end{array}$ & HaR2a ou HaR2b \\
\hline $\begin{array}{l}\text { "A taxa de juros depende do desejo por } \\
\text { reservas líquidas e do montante de } \\
\text { moeda disponível para satisfazer este } \\
\text { desejo" }\end{array}$ & $\mathrm{r}=\mathrm{f}_{10}\left(\mathrm{~L}, \mathrm{M}^{\mathrm{S}}\right)$ & HaR3 \\
\hline $\begin{array}{l}\text { "Este montante de moeda disponível } \\
\text { depende da política dos bancos ao } \\
\text { determinarem o valor de suas dívidas a } \\
\text { receber e do nível de renda (quanto } \\
\text { maior o nível de renda, mais moeda será } \\
\text { necessária para a circulação ativa)" }\end{array}$ & $\mathrm{M}^{\mathrm{S}}=\mathrm{f}_{11}(\mathrm{~b}, \mathrm{Y})$ & HaR4 \\
\hline
\end{tabular}

Fonte: HARROD, 1937, p. 79.

Neste sistema, dados a eficiência marginal do capital, a propensão a consumir, a preferência pela liquidez e a oferta de moeda, determina-se o valor das incógnitas investimento, renda e taxa de juros. Mas, comparando-se este sistema resumido com o atribuído à "teoria de Keynes" descrito no quadro 2, nota-se que:

(I) HARROD recorre a uma nova "transposição" e a uma "ampliação", não explícitas, da primeira equação: inverte a relação entre investimento e eficiência marginal do capital, passando de uma relação de causalidade que definia o investimento como determinante da eficiência marginal do capital $\left[e m c=\mathrm{f}_{1}(\mathrm{I})\right.$ em $\mathrm{HaC} 1$ e em $\mathrm{HaK} 1$ ], para uma relação inversa e mais completa, segundo a qual o investimento é que é determinado não apenas pela eficiência marginal do capital como também pela taxa de juros $\left[\mathrm{I}=\mathrm{f}_{9}(\mathrm{emc}, \mathrm{r})\right.$ em HaR1]. Consequentemente, neste passo do seu raciocínio, HARROD abandona o pressuposto da igualdade entre eficiência marginal do capital e taxa de juros.

(II) HARROD não é explícito quanto à inclusão ou não da taxa de 
juros como determinante da renda - suposição que reconhece não corresponder à formulação original de Keynes. Assim, as equações HaR2a e HaR2b correspondem, respectivamente, às equações HaK2b e HaK2c. A transposição que se iniciava na equação HaK2a (na qual a poupança é determinada pela taxa de juros e pelo nível de renda) desaparece do argumento.

(III) a oferta de moeda, que era considerada conhecida e dependente da política monetária passa a depender da renda e da política dos bancos (incógnita b na equação HaR4).

(IV) A taxa de juros, que dependia da oferta de moeda (dada) e da renda, passa a depender da oferta de moeda e da preferência pela liquidez. ${ }^{29}$

Das observações anteriores, a mais importante é a última. HARROD corrige, por mera substituição, o erro de notação que havia cometido ao escrever $\mathrm{y}=\chi(\mathrm{m}, \mathrm{i})$ [ou $\mathrm{r}=\mathrm{f}_{7}\left(\mathrm{M}^{\mathrm{S}}, \mathrm{Y}\right)$ ] na equação $\mathrm{HaK} 3 \mathrm{~b}$, quando argumentava que a inclusão da renda como determinante da taxa de juros decorria do fato de que "o montante de moeda requerido pelos consumidores e comerciantes para a circulação ativa depende do nível de renda" (HARROD, 1937, p. 78). Ou seja, a equação HaK3b é substituída pela equação HaR3 representada por $\mathrm{r}=\mathrm{f}_{10}(\mathrm{~L}$, $\mathrm{M}^{\mathrm{S}}$ ). Mas, conforme assinalado anteriormente, a notação correta seria $\mathrm{M}^{\mathrm{D}}{ }_{\mathrm{T}}=$ $\mathrm{f}_{8}(\mathrm{Y})$. HARROD de fato adiciona ao seu sistema uma equação de natureza semelhante, na qual considera também o nível de preços. Para fazê-lo, propõe uma nova formulação para a determinação da taxa de juros, partindo da consideração de que a demanda por moeda não se resume ao motivo "transação" e sugerindo que a demanda por moeda para os motivos "especulação" e "precaução" seja vista como residual, isto é, "para outros fins que não os da circulação ativa." ${ }^{30}$ (HARROD, 1937, p. 83, n. 1)

Segundo HARROD, como o montante de moeda disponível para reservas líquidas é limitado e não pode ser ampliado por mero desejo do público, a renda esperada das reservas menos líquidas (juros) deve ser tal que induza o público a abrir mão da liquidez ou a "restringir os que desejam manter liquidez

29 Se em HaR4 a "política dos bancos" fosse representada por r, a taxa de juros, considerando a observação (III) acima, concluiría-se que a taxa de juros depende da oferta de moeda (que por sua vez depende da taxa de juros e da renda) e da preferência pela liquidez. Formalmente, tería-se algo como $\mathrm{r}=\mathrm{f}_{10}\left\{\mathrm{f}_{11}(\mathrm{r}, \mathrm{Y}), \mathrm{L}\right\}$.

30 “O resíduo de moeda, não demandado para a circulação ativa, está disponível para as pessoas comuns, desencorajadas por seus corretores a investirem imediatamente e, mais importante, para as firmas que queiram manter liquidez para ampliação do capital ou outros objetivos semelhantes no curto espaço de seis meses a dois anos, e que não queiram manter suas reservas na forma de títulos que envolvem algum risco de depreciação durante este período." (HARROD, 1937, p. 78) 
ao montante de moeda disponível para este fim" (HARROD, 1937, p. 78). Isto explicaria porque, quanto menor for a quantidade de moeda disponível, maior será a taxa de juros. Assim, no lugar da "oferta de moeda" que determina a taxa de juros [equação HaR3], HARROD propõe que se utilize a quantidade de moeda disponível e residual, isto é, a diferença entre a quantidade total de moeda existente e aquela necessária para a circulação ativa. Em termos da notação proposta, a equação HaR3 $\left[\mathrm{r}=\mathrm{f}_{10}\left(\mathrm{~L}, \mathrm{M}^{\mathrm{S}}\right)\right]$ assume a forma $\mathrm{r}=\mathrm{f}_{15}\left(\mathrm{~L}, \mathrm{M}_{\mathrm{E}}^{\mathrm{D}}+\mathrm{M}_{\mathrm{p}}^{\mathrm{D}}\right){ }^{31}$

Além disso, HARROD considera que a demanda por moeda para transação depende não apenas do nível de atividade (ou renda), mas também do nível de preços. Esta relação poderia ser descrita por $\mathrm{M}_{\mathrm{T}}^{\mathrm{D}}=\mathrm{f}_{14}(\mathrm{Y}, \mathrm{p})$. $\mathrm{O}$ nível de preços, por sua vez, é uma função do próprio nível de atividade, não no sentido da teoria quantitativa da moeda, mas no sentido dado por Keynes, segundo quem, "o nível geral de preços é completamente flexível e determinado pelas equações do campo geral (da teoria econômica) sem referência à quantidade de moeda" (HARROD, 1936, p. 80) - mas dependente dos custos (marginais) de produção. ${ }^{32}$ Segundo HARROD, os fatores de produção a serem levados em conta são o capital, a moeda e o trabalho. Como o capital já está considerado

31 Já que a quantidade de moeda disponível para ser demandada pelos motivos especulação e precaução é o total de moeda descontado, o montante necessário para a circulação ativa, ou seja, MDE + MDP = MS - MDT. Isto implica, naturalmente, que HARROD considera a condição de igualdade entre demanda (total) de moeda e oferta de moeda, isto é, MDTOTAL = MS. Veja o argumento completo do autor: "Em sua equação da preferência pela liquidez, Keynes inclui a demanda por moeda para qualquer propósito, e a quantidade de moeda que aparece nela é a quantidade total de moeda da comunidade. Pareceu ser mais simples nesta parte da exposição dividir este total em duas partes, o montante necessário para a circulação ativa e o resíduo, para definir a quantidade de moeda que aparece na equação de preferência pela liquidez como este resíduo, e a demanda que a equação expressa como a demanda para outros fins que não os da circulação ativa. Esta redefinição de termos é meramente um instrumento de exposição e não implica qualquer ruptura com a doutrina essencial do Sr. Keynes." (HARROD, 1937, p. 83, n. 1)

32 Para HARROD, a questão da determinação do nível geral de preços constitui uma diferença crucial entre a teoria tradicional e a teoria de Keynes: "na teoria tradicional, o nível de preços é determinado pela equação [quantitativa] da moeda [...] enquanto que na teoria de Keynes, o nível de preços é determinado pelo custo monetário da produção, com as modificações apropriadas para [considerar-se] a concorrência imperfeita" (HARROD, 1937, p. 82). O "campo geral da teoria econômica" a que esta passagem se refere é a parte da teoria que permite considerar-se como variáveis elementos que são tomados como dados nos "ramos especializados". Vale ressaltar que HICKS e MEADE também consideram, em seus respectivos sistemas representativos da "teoria de Keynes", um conjunto de equações que descrevem este “campo geral", no qual os preços não são dados e são determinados pelos custos marginais de produção, independentemente da quantidade de moeda. 
na equação do investimento e a moeda na equação da taxa de juros, é preciso apenas incluir a taxa nominal de salários. Assim, a notação proposta seria $\mathrm{p}=$ $\mathrm{f}_{12}(\mathrm{w}, \mathrm{Y}){ }^{33}$

A interpretação de HARROD, de que a demanda por moeda pelos motivos "especulação" e "transação" é uma demanda residual, de que a demanda por moeda pelo motivo "transação" depende do nível de renda e do nível de preços e sua concepção de que o nível de preços depende do próprio nível de renda, resguarda "a mútua interdependência de todo o sistema, ao mesmo tempo em que preserva os 'atalhos' necessários para se pensar sobre problemas específicos" (HARROD, 1937, p. 83). Em outras palavras, a teoria de Keynes pode ser sistematizada por um conjunto de equações simultâneas, mutuamente interdependentes, que permitem relacionar não apenas as três incógnitas (renda, investimento e taxa de juros), mas também incorporar o nível de preços. Por este motivo, o sistema de equações que leva em conta estas últimas observações pode ser denominado de "teoria de Keynes generalizada".

\section{CONCLUSÃo}

Segundo HARROD, o sistema de Keynes corrige algumas características insatisfatórias da teoria tradicional. A primeira delas refere-se ao fato de permitir que o nível de renda não seja tomado como dado nas equações dos "ramos especializados", mas, pelo contrário, que seja determinado por meio dessas equações. A segunda característica corrigida pelo sistema de Keynes diz respeito à determinação do nível de preços, que deixa de depender da equação quantitativa da moeda e leva em conta o custo de produção. A terceira característica corrigida é a explicitação da distribuição da demanda por moeda entre a demanda requerida para a circulação ativa (que depende da renda) e a demanda "residual", que é função da taxa de juros.

O sistema de Keynes, segundo HARROD, é, portanto, superior ao da teoria tradicional. Constitui-se num sistema interdependente, segundo o qual:

[...] o montante do investimento depende da produtividade marginal do capital e da taxa de juros. O nível de renda e de atividade está relacionado ao montante de investimento pelo multiplicador, isto é,

33 Vale destacar que HARROD adota o pressuposto de que a taxa nominal de salários é determinada no mercado de trabalho, e que enfatiza a impossibilidade da barganha salarial se dar em termos reais. Ver HARROD (1937, p. 80). 
pela propensão marginal a consumir; o nível de preços está relacionado ao nível de atividade pelo custo monetário de produção (que depende do montante de atividade realizada); o montante de moeda absorvida pelas transações ativas depende do volume das transações e do nível de preços, e o montante de moeda para reservas líquidas é igual ao montante total de moeda no sistema descontado o montante requerido para a circulação ativa; a taxa de juros depende do montante de moeda disponível para reservas líquidas e as escalas de preferência pela liquidez. (HARROD, 1937, p. 83-84)

Estas relações finais também podem ser formalizadas. O quadro 4 a seguir apresenta o conjunto final de equações que representam a "teoria de Keynes generalizada" na interpretação de HARROD. ${ }^{34}$ Como o quadro 3, o quadro 4 formaliza, com símbolos matemáticos, os argumentos verbais de HARROD, enquanto os quadros 1 e 2 apenas organizam sua formalização original. Todos eles foram construídos para ressaltar a importância de se relacionar a formulação matemática aos argumentos discursivos (e vice-versa).

Assim, é importante notar que a equação HaG1 é igual à equação HaR1, e se origina de uma inversão e ampliação da equação HaK1. A equação HaG2 corresponde à equação HaK2c, que é uma versão simplificada da equação HaK2b, que por sua vez é a versão transposta da equação HaK2a. ${ }^{35}$ As equações HaG3 e HaG4 constituem a inclusão, no sistema, de elementos que costumam pertencer à "parte geral" da teoria econômica, relativa à determinação dos preços e da taxa salarial. A equação HaG5 é uma ampliação da equação HaK3c - que não aparece de forma explícita no quadro 3. A equação HaG6 é definicional e corresponde à distribuição da demanda por moeda entre os motivos transação, precaução e especulação, com a condição implícita de que a demanda por moeda iguala a oferta de moeda. Por fim, a equação HaG7 é uma reformulação, com base na definição anterior, da equação HaR3, que por sua vez modifica radicalmente a equação HaK3b.

34 Como no quadro 3, a formalização matemática não consta do original.

35 Note que a equação HaK2b se repete na HaR2a e a equação HaK2c na HaR2b. 
Quadro 4 - TEORIA DE KEYNES GENERALIZADA

\begin{tabular}{|c|c|c|}
\hline & notação sugerida & $\begin{array}{l}\text { Ha: HARROD } \\
\text { G: Keynes } \\
\text { Generalizado }\end{array}$ \\
\hline $\begin{array}{l}\text { "O montante de investimento depende } \\
\text { da produtividade marginal do capital e } \\
\text { da taxa de juros" }\end{array}$ & $\begin{array}{l}\mathrm{I}=\mathrm{f}_{9}(\mathrm{emc}, \mathrm{r}) \\
\mathrm{f}_{5} \text { representa a propensão } \\
\text { marginal a consumir }\end{array}$ & HaG1 \\
\hline $\begin{array}{l}\text { "O nível de renda e de atividade está } \\
\text { relacionado ao montante de } \\
\text { investimento pelo multiplicador, isto é, } \\
\text { pela propensão marginal a consumir" }\end{array}$ & $\mathrm{Y}=\mathrm{f}_{5}(\mathrm{I})$ & $\mathrm{HaG} 2$ \\
\hline $\begin{array}{l}\text { "o nível de preços está relacionado ao } \\
\text { nível de atividade pelo custo monetário } \\
\text { de produção (que depende do montante } \\
\text { de atividade realizada)" }\end{array}$ & $\begin{array}{c}\mathrm{p}=\mathrm{f}_{12}(\mathrm{w}, \mathrm{Y}) \\
\mathrm{w}=\mathrm{f}_{13}(\mathrm{Y})\end{array}$ & $\begin{array}{l}\mathrm{HaG} 3 \\
\mathrm{HaG} 4\end{array}$ \\
\hline $\begin{array}{l}\text { "o montante de moeda absorvida pelas } \\
\text { transações ativas depende do volume } \\
\text { das transações e do nível de preços" }\end{array}$ & $\mathrm{M}_{\mathrm{T}}^{\mathrm{D}}=\mathrm{f}_{14}(\mathrm{Y}, \mathrm{p})$ & HaG5 \\
\hline $\begin{array}{l}\text { "o montante de moeda para reservas } \\
\text { líquidas é igual ao montante total de } \\
\text { moeda no sistema descontado o } \\
\text { montante requerido para a circulação } \\
\text { ativa" }\end{array}$ & $\begin{array}{l}\mathrm{M}_{\mathrm{E}}^{\mathrm{D}}+\mathrm{M}_{\mathrm{P}}^{\mathrm{D}}=\mathrm{M}^{\mathrm{S}}-\mathrm{M}_{\mathrm{T}}^{\mathrm{D}} \\
\text { Observe-se que } \mathrm{M}^{\mathrm{D}} \\
=\mathrm{M}_{\mathrm{E}}^{\mathrm{D}}+\mathrm{M}_{\mathrm{P}}^{\mathrm{D}}+\mathrm{M}_{\mathrm{T}}^{\mathrm{D}}=\mathrm{M}^{\mathrm{S}}\end{array}$ & HaG6 \\
\hline $\begin{array}{l}\text { "a taxa de juros depende do montante } \\
\text { de moeda disponível para reservas } \\
\text { líquidas e as escalas de preferência pela } \\
\text { liquidez" }\end{array}$ & $\begin{array}{c}\mathrm{r}=\mathrm{f}_{15}\left(\mathrm{~L}, \mathrm{M}_{\mathrm{E}}^{\mathrm{D}}+\mathrm{M}_{\mathrm{P}}^{\mathrm{D}}\right)= \\
\mathrm{f}_{15}\left(\mathrm{~L}, \mathrm{M}_{\text {TOTAL }}^{\mathrm{D}}-\mathrm{M}_{\mathrm{T}}^{\mathrm{D}}\right)\end{array}$ & HaG7 \\
\hline
\end{tabular}

Fonte: HARROD, 1937, p. 83-84.

Quando estas relações não são feitas com o cuidado necessário, elas originam interpretações como, por exemplo, a de YOUNG (1987), para quem "HARROD construiu um sistema no qual a poupança é determinada pela renda e pela taxa de juros, e a taxa de juros é determinada pela preferência pela liquidez, pela oferta de moeda e pelo nível de renda" (YOUNG, 1987, p. 22). Ou seja, na equação de poupança atribuída a HARROD por YOUNG $[S=S(Y, r)]$, não se considera o raciocínio desenvolvido originalmente pelo autor em questão descrito no quadro 2 pelas equações HaK2a, HaK2b e HaK2c. Além disso, na interpretação de YOUNG, para HARROD a renda é um dos determinantes da taxa de juros $\left[r=r\left(L, M^{S}, Y\right)\right]-$ mas isto não encontra sustentação no artigo original. Embora HARROD tenha de fato escrito com símbolos matemáticos 
que a taxa de juros depende da quantidade de moeda e do nível de renda, foi aqui ressaltado anteriormente que sua notação não corresponde ao seu argumento verbal e demonstrado como HARROD refaz seu argumento. Ainda considerando a análise de YOUNG, deve-se reconhecer que este intérprete tem razão em destacar que HARROD construiu um sistema de equações "interdependentes e de natureza reversível" (YOUNG, 1987, p. 24) - neste sentido semelhante ao de HICKS (e outros). Mas o sistema de equações que HARROD apresenta efetivamente no seu texto é mais pobre do que o conjunto de argumentos e raciocínios que desenvolve para descrever as principais relações de causalidade que representam a sua visão do que vem a ser a essência da Teoria Geral de Keynes. Neste sentido, a conclusão de YOUNG, de que a formalização de HARROD é a fonte de inspiração de HICKS, passa a ser bastante questionável, não apenas devido ao empobrecimento da interpretação de YOUNG, mas também e principalmente porque a formalização de HICKS (e os seus argumentos) não corresponde à de HARROD. Mas o detalhamento deste tema é o que vem sendo formulado no escopo da pesquisa ainda inconclusa a que se fez menção no início deste trabalho. ${ }^{36}$

\section{RESUMO}

A hipótese discutida na pesquisa (ainda inconclusa) que dá origem a este texto é a de que as formalizações matemáticas da Teoria Geral do Emprego, dos Juros e da Moeda, elaboradas por Roy HARROD, John HICKS e James MEADE, além das de David CHAMPERNOWNE e de Brian REDDAWAY, ainda que semelhantes na forma final, foram alcançadas mediante diferentes raciocínios, justificativas e argumentos teóricos, e que a aceitação e o sucesso da versão matematizada se deu pelo fato dela permitir a incorporação - de modo implícito - das variadas relações de causalidade definidas por cada um destes autores. Por razões de tempo e de espaço, o texto explora esta hipótese de forma ainda preliminar e cinge-se apenas à contribuição de HARROD.

36 As análises do texto de HICKS (1937), feitas por YOUNG (1987), DARITY e YOUNG (1995) e BARENS (1998), sofrem dos mesmos tipos de problemas que se acabou de descrever com relação ao trabalho de HARROD (1937). 


\begin{abstract}
The hypothesis behind the ongoing research that motivates this paper suggests that the mathematical formalizations of the General Theory of Employment, Interest and Money advanced by Roy HARROD, John HICKS and James MEADE, as well as the ones by David CHAMPERNOWNE and Brian REDDAWAY, although similar in their form, were constructed using different kinds of reasonings, justifications and theoretical arguments. Consequently, the acceptance and success of the mathematized version occurred because it allowed the implicit - incorporation of the diverse causal relations proposed by each of these authors. Lack of space and time prevent a complete development of this hypothesis here, so this text focuses only on HARROD's contribution.
\end{abstract}

\title{
REFERÊNCIAS
}

BARENS, I. From Keynes to Hicks - an aberration? IS-LM and the analytical nucleus of the general theory. Paper presented at the Second Annual Conference of the European Society for the History of Economic Thought, Bologna, 27/02-1/03/1998. Mimeog. To be published in ANTONI, E. di; HOWITT, P.; LEIJONHUFVUD, A. (Ed.). Money, markets and method: essays in honor of Robert Clower. Chletenhna: Edward Elgar, 1998.

BEAUD, M.; DOSTALER, G. Economic thought since Keynes - a history and dictionary of major economists. London: Routledge, 1995.

CHAMPERNOWNE, D. G. Unemployment, basic and monetary: the classical analysis and the keynesian. Review of Economic Studies, v. 3, p. 201-216, July 1936.

DARITY JR., W.; YOUNG, W. IS-LM: an inquest. History of Political Economy, v. 27, n. 1, p. 1-41, 1995.

HARROD, R. F. Review of the general theory of employment, interest and money. Political Quarterly, v. 7, n. 1/4, p. 293-298, Apr. 1936.

HARROD, R. F. Mr. Keynes and traditional theory. Econometrica, v. 5, p. 74-86, Jan. 1937.

HICKS, J. R. The General Theory: a first impression. In: Money, interest and wages collected essays in economic theory. Oxford: Blackwell, 1936. v. 1, p. 83-99.

Mr. Keynes and the 'classics': a suggested interpretation. Econometrica, v. 5, p. 147159, Apr. 1937. 
MEADE, J. E. A simplified model of Mr. Keynes' system. Review of Economic Studies, v. 4, p. 98-107, Feb. 1937.

MOGGRIDGE, D. (Ed.). John Maynard Keynes: the General Theory and after - Part II: defence and development. London: Macmillan, 1973/1987. (The collected writings of John Maynard Keynes-CWJMK, 14).

O'DONNELL, R. Keynes and formalism. In: HARCOURT, G. C.; RIACH, R. A. A 'second edition' of the General Theory. London: Routledge, 1997a. v. 2, p. 131-165.

PATINKIN, D. On different interpretations of 'The General Theory'. Journal of Monetary Economics, v. 26, p. 205-243, Oct. 1990.

PHELPS-BROWN, E. H. Report of the Oxford Meeting, September 25-29 1936. Econometrica, v. 5, p. 361-383, Oct. 1990.

REDDAWAY, W. B. The General Theory of employment, interest and money. Economic Record, v. 12, p. 28-36, June 1936.

SKIDELSKY, R. John Maynard Keynes - the economist as saviour 1920-1937. London: Macmillan, 1992.

YOUNG, W. Interpreting Keynes: The IS/LM Enigma. Boulder, Colorado: Westview Press; Oxford: Basil Blackwell, 1987.

\section{APÊNDICE: LISTA DAS EQUAÇÕES}

\section{Quadro 1 - TEORIA TRADICIONAL, NOTAÇÃO ORIGINAL}

\begin{tabular}{|c|c|c|}
\hline & notação alternativa & $\begin{array}{l}\text { Ha: HARROD } \\
\text { C: Clássicos }\end{array}$ \\
\hline $\begin{array}{l}\text { "a produtividade marginal do capital } \\
\text { depende do montante de capital } \\
\text { investido por unidade de tempo." } \\
\text { (HARROD, } 1937, \text { p. } 76 \text { ) }\end{array}$ & emc $=f_{1}(I)$ & $\mathrm{HaCl}$ \\
\hline $\begin{array}{l}\text { "O montante que as pessoas escolhem } \\
\text { poupar, que é igual ao montante de } \\
\text { investimento, depende da taxa de } \\
\text { juros." (HARROD, } 1937, \text { p. } 76 \text { ) }\end{array}$ & $\begin{array}{c}\mathrm{S}=\mathrm{f}_{2}(\mathrm{r}) \text { ou } \mathrm{I}=\mathrm{f}_{2}(\mathrm{r}) \\
\text { pois } \mathrm{I}=\mathrm{S} \text { por definição }\end{array}$ & $\mathrm{HaC} 2$ \\
\hline $\begin{array}{l}\text { "investe-se capital até que sua } \\
\text { produtividade marginal iguale a taxa de } \\
\text { juros." (HARROD, 1937, p. 76) }\end{array}$ & $\mathrm{emc}=\mathrm{r}$ & $\mathrm{HaC} 3$ \\
\hline
\end{tabular}

Fonte: HARROD, 1937. 
Quadro 2 - TEORIA DE KEYNES, NOTAÇÃO ORIGINAL

\begin{tabular}{|c|c|c|}
\hline & notação alternativa & $\begin{array}{c}\text { Ha: HARROD } \\
\text { K: Keynes }\end{array}$ \\
\hline $\begin{array}{l}\text { "a eficiência marginal do capital é uma } \\
\text { função do investimento" (HARROD, } \\
\text { 1937, p. 76), sem considerar, na } \\
\text { formulação, que "o valor [da } \\
\text { eficiência marginal] depende das } \\
\text { expectativas empresariais" (HARROD, } \\
\text { 1937, p. 77). Portanto, a equação HaC1 } \\
\text { é igual à equação HaK1. }\end{array}$ & $\mathrm{emc}=\mathrm{f}_{1}(\mathrm{I})$ & HaK1 \\
\hline $\begin{array}{l}\text { "o montante de poupança depende não } \\
\text { apenas da taxa de juros, mas também } \\
\text { do nível de renda da comunidade" } \\
\text { (HARROD, 1937, p. 77) transposta } \\
\text { para "o nível de renda depende do } \\
\text { montante de investimento (= poupança) } \\
\text { e da taxa de juros" (HARROD, 1937, } \\
\text { p. 77) simplificada para "[Keynes] caiu } \\
\text { na tentação de perder a taxa de juros } \\
\text { de vista [na doutrina do multiplicador] } \\
\text { para o bem de um atalho ainda mais } \\
\text { curto." (HARROD, 1937, p. 78) }\end{array}$ & $\begin{array}{c}\mathrm{S}=\mathrm{f}_{3}(\mathrm{r}, \mathrm{Y}) \\
\text { transposta para } \\
\mathrm{Y}=\mathrm{f}_{4}(\mathrm{I}, \mathrm{r}) \text { ou } \mathrm{Y}=\mathrm{f}_{4}(\mathrm{~S}, \mathrm{r}) \\
\text { simplificada para } \\
\mathrm{Y}=\mathrm{f}_{5}(\mathrm{I}) \text { ou } \mathrm{Y}=\mathrm{f}_{5}(\mathrm{~S})\end{array}$ & HaK2b \\
\hline $\begin{array}{l}\text { "este é o esquema da preferência pela } \\
\text { liquidez [...] em que m, a quantidade } \\
\text { de moeda, é um termo conhecido e } \\
\text { depende da política monetária" } \\
\text { (HARROD, 1937, p. 78) ampliada para } \\
\text { "o nível de renda deve ser incluído na } \\
\text { equação, uma vez que o montante de } \\
\text { moeda requerido pelos consumidores } \\
\text { e comerciantes para a circulação ativa } \\
\text { depende do nível de renda" } \\
\text { (HARROD, 1937, p. 78) corrigida para } \\
\text { "o montante de moeda requerido pelos } \\
\text { consumidores e comerciantes para a } \\
\text { circulação ativa depende do nível de } \\
\text { renda." (HARROD, 1937, p. 78) }\end{array}$ & $\begin{array}{l}\text { ampliada para } \\
\mathrm{r}=\mathrm{f}_{7}\left(\mathrm{M}^{\mathrm{S}}, \mathrm{Y}\right) \\
\text { corrigida para } \\
\mathrm{M}_{\mathrm{T}}^{\mathrm{D}}=\mathrm{f}_{8}(\mathrm{Y})\end{array}$ & $\mathrm{HaK} 3 \mathrm{c}$ \\
\hline $\begin{array}{l}\text { "investe-se capital até que sua } \\
\text { produtividade marginal iguale a taxa de } \\
\text { juros." (HARROD, 1937, p. 76) }\end{array}$ & $\mathrm{emc}=\mathrm{r}$ & HaK4 \\
\hline
\end{tabular}

Fonte: HARROD, 1937. 
Quadro 3 - TEORIA DE KEYNES (Resumo)

\begin{tabular}{|l|c|c|}
\hline & notação sugerida & $\begin{array}{c}\text { Ha: HARROD } \\
\text { R: Keynes } \\
\text { Resumido }\end{array}$ \\
\hline $\begin{array}{l}\text { "O montante de investimento (= montante } \\
\text { de poupança) depende da produtividade } \\
\text { marginal do capital e da taxa de juros" }\end{array}$ & $\mathrm{I}=\mathrm{f}_{9}(\mathrm{emc}, \mathrm{r})$ & HaR1 \\
\hline $\begin{array}{l}\text { "Onível de renda está relacionado ao montante } \\
\text { de investimento através do multiplicador, isto } \\
\text { é, pela propensão a consumir" }\end{array}$ & $\begin{array}{c}\mathrm{Y}=\mathrm{f}_{4}(\mathrm{I}, \mathrm{r}) \text { ou } \mathrm{Y}=\mathrm{f}_{5}(\mathrm{I}) \\
\mathrm{f}_{5} \text { representa a propensão } \\
\text { marginal a consumir }\end{array}$ & HaR2a ou HaR2b \\
\hline $\begin{array}{l}\text { "A taxa de juros depende do desejo por } \\
\text { reservas líquidas e do montante de moeda } \\
\text { disponível para satisfazer este desejo" }\end{array}$ & $\mathrm{r}=\mathrm{f}_{10}\left(\mathrm{~L}, \mathrm{M}^{\mathrm{s}}\right)$ & HaR3 \\
\hline $\begin{array}{l}\text { "Este montante de moeda disponível depende } \\
\text { da política dos bancos ao determinaremo valor } \\
\text { de suas dívidas a receber e do nível de renda } \\
\text { (quanto maior o nível de renda, mais moeda } \\
\text { será necessária para a circulação ativa)" }\end{array}$ & $\mathrm{M}^{\mathrm{s}}=\mathrm{f}_{11}(\mathrm{~b}, \mathrm{Y})$ & HaR4 \\
\hline
\end{tabular}

Fonte: HARROD, 1937, p. 79.

Quadro 4 - TEORIA DE KEYNES GENERALIZADA

\begin{tabular}{|c|c|c|}
\hline & notação sugerida & $\begin{array}{l}\text { Ha: HARROD } \\
\text { G: Keynes } \\
\text { Generalizado } \\
\end{array}$ \\
\hline $\begin{array}{l}\text { "O montante de investimento depende da } \\
\text { produtividade marginal do capital e da taxa } \\
\text { de juros" }\end{array}$ & $\mathrm{I}=\mathrm{f}_{9}(\mathrm{emc}, \mathrm{r})$ & HaG1 \\
\hline $\begin{array}{l}\text { "O nível de renda e de atividade está } \\
\text { relacionado ao montante de investimento } \\
\text { pelo multiplicador, isto é, pela propensão } \\
\text { marginal a consumir" }\end{array}$ & $\begin{array}{c}\mathrm{Y}=\mathrm{f}_{5}(\mathrm{I}) \\
\mathrm{f}_{5} \text { representa a } \\
\text { propensão marginal a } \\
\text { consumir }\end{array}$ & $\mathrm{HaG} 2$ \\
\hline $\begin{array}{l}\text { "O nível de preços está relacionado ao nível de } \\
\text { atividade pelo custo monetário de produção (que } \\
\text { depende do montante de atividade realizada)" }\end{array}$ & $\begin{array}{l}\mathrm{p}=\mathrm{f}_{12}(\mathrm{w}, \mathrm{Y}) \\
\mathrm{w}=\mathrm{f}_{13}(\mathrm{Y})\end{array}$ & $\begin{array}{l}\text { HaG3 } \\
\text { HaG4 }\end{array}$ \\
\hline $\begin{array}{l}\text { "O montante de moeda absorvida pelas } \\
\text { transações ativas depende do volume das } \\
\text { transações e do nível de preços" }\end{array}$ & $\mathrm{M}_{\mathrm{T}}^{\mathrm{D}}=\mathrm{f}_{14}(\mathrm{Y}, \mathrm{p})$ & HaG5 \\
\hline $\begin{array}{l}\text { "O montante de moeda para reservas } \\
\text { líquidas é igual ao montante total de moeda } \\
\text { no sistema descontado o montante } \\
\text { requerido para a circulação ativa" }\end{array}$ & $\begin{array}{c}\mathrm{M}_{\mathrm{E}}^{\mathrm{D}}+\mathrm{M}_{\mathrm{P}}^{\mathrm{D}}=\mathrm{M}^{\mathrm{S}}-\mathrm{M}_{\mathrm{T}}^{\mathrm{D}} \\
\text { Note-se que } \\
\mathrm{M}^{\mathrm{S}}=\mathrm{M}^{\mathrm{D}}{ }_{\text {TOTAL }}= \\
\mathrm{M}_{\mathrm{E}}^{\mathrm{D}}+\mathrm{M}_{\mathrm{P}}^{\mathrm{D}}+\mathrm{M}_{\mathrm{T}}^{\mathrm{D}}\end{array}$ & HaG6 \\
\hline $\begin{array}{l}\text { "a taxa de juros depende do montante de } \\
\text { moeda disponível para reservas líquidas e } \\
\text { as escalas de preferência pela liquidez" }\end{array}$ & $\begin{array}{l}\mathrm{r}=\mathrm{f}_{15}\left(\mathrm{~L}, \mathrm{M}_{\mathrm{E}}^{\mathrm{D}}+\mathrm{M}_{\mathrm{P}}^{\mathrm{D}}\right)= \\
\mathrm{f}_{15}\left(\mathrm{~L}, \mathrm{M}_{\text {TOTAL }}^{\mathrm{D}}-\mathrm{M}_{\mathrm{T}}^{\mathrm{D}}\right)\end{array}$ & $\mathrm{HaG7}$ \\
\hline
\end{tabular}

Fonte: HARROD, 1937, p. 83-84. 
